SCHWERPUNKT

\section{Digital Rights Management - Herausforderungen für Recht, Technik und Technikfolgenabschätzung}

\section{Eine Einführung in den Schwerpunkt}

\section{von Knud Böhle und Carsten Orwat, ITAS}

\section{DRM ist Realität}

Ob bewusst oder nicht, mit Techniken des Digital Rights Managements (DRM) haben die meisten schon zu tun gehabt: DVDs sind mit einem Kopierschutz und zusätzlich einem „regional code“ versehen, der z. B. verhindert, dass eine in den USA gekaufte DVD in Deutschland abgespielt werden kann. Auch bei CDs werden zunehmend DRM-Systeme eingesetzt. Bereits 2003 waren von den Top Ten der CD-Alben sieben mit technischem Kopierschutz versehen (Hansen 2003, S. 136). ${ }^{1}$ Online-Musikläden, deren bekanntester vielleicht „iTunes“ und deren meistgenutzter in Deutschland laut Brenner-Studie (GfK/ BPW 2006) „musicload“ ist, liefern die angebotenen Musikstücke meistens in DRM-geschützten Formaten, und ähnliches gilt für Musikangebote für das Handy. Zu den Anwendungen jenseits des Unterhaltungsbereichs, die vielleicht weniger bekannt sind, gehören etwa die Elektronischen Dokumentlieferdienste (wie „subito“ in Deutschland) oder die Ausleihsysteme von Bibliotheken, die es erlauben, elektronische Bücher über das Internet befristet zugänglich $\mathrm{zu}$ machen. ${ }^{2}$ Zwar sind es derzeit hauptsächlich die großen Medienunternehmen und die Informationsvermittler, die DRM-Systeme einsetzen, um Zugang und Nutzung digitaler Inhalte zu kontrollieren. Aber mit der Zunahme nichtkommerzieller Angebote im Internet („Creati- ve Commens“-lizensierte Inhalte, „Open Access Repositories") stellt sich auch für deren Urheber und Anbieter die Frage, wie und in welchem Umfang die Nutzung dieser Angebote in ihrem eigenen Interesse zu kontrollieren ist. Um den Gedanken der möglichen Dezentralisierung der Kontrolle noch weiter zu verdeutlichen: In gewisser Weise setzen viele von uns schon heute DRM-Funktionalität ein, wenn sie z. B. bestimmte Sicherheitseinstellungen (z. B. „Drucken nicht erlaubt" und „Text entnehmen nicht erlaubt") des „Adobe Acrobat" verwenden, bevor sie Texte an andere weitergeben.

\section{DRM-Systeme als Kontrolltechnologie}

Computertechnologie kam zunächst als Kontrolltechnik par excellence ins Spiel. ${ }^{3}$ In den 1990er Jahren und nicht zuletzt durch das Internet wurde dann der Doppelcharakter des Computers als Maschine und Medium deutlich. Damals diagnostizierte Shapiro erneut eine ,control revolution“ in seinem Buch gleichen Titels (1999) - nun auf den Computer als Medium bezogen: „....the Net's richest potential feature [is]: individuals will exercise more control over the flow of information, and over the way that society understands issues and, ultimately, itself" (S. 43). Anders als bei den traditionellen analogen Medien gibt es hier keine mit den Materialeigenschaften der Trägermedien (Papier, Magnetband etc.) einhergehende, implizite Kontrollemechanismen mehr, die Kopieren und Verbreiten (Zeitaufwand, Kosten, Qualitätsverlust) beschränken könnten. Im Gegenteil: Die neue technische Infrastruktur, die durch die offene Architektur des Internet, offene Formate (wie MP3) und im Prinzip offene Endgeräte wie den PC gekennzeichnet ist, erlaubt von ihren ,Materialeigenschaften" her geradezu das perfekte, kostengünstige und unbegrenzte Vervielfältigungen von Inhalten bzw. digitalen Objekten jeder Art und schnellste weltweite Verbreitung - zunächst unabhängig davon, ob diese Handlungen legal oder illegal sind.

Diese neue Lage wurde von den Medienunternehmen als Kontrollverlust wahrgenommen, und es entstand der Wunsch der Urheber und mehr noch der Verwerter, das Geschehen 
besser zu kontrollieren. Das sollte durch explizite technische Kontrollvorkehrungen (,technical controls“) erreicht werden. Durch sie sollten nicht erlaubte Nutzungen geistigen Eigentums unterbunden werden. Gleichzeitig sollte damit eine nun „künstliche Knappheit“" hergestellt werden, die für das Funktionieren von Märkten für digitale Medienangebote und Informationsgüter als notwendig erachtet wurde. Obwohl Kontrolle häufig mit negativen Assoziationen verbunden ist, ist technische Kontrolle auch die Bedingung für neue Möglichkeiten - in unserem Kontext etwa Möglichkeiten erhöhter Produktdifferenzierung und neuer Dienstleitungen im Internet.

Das führt uns $\mathrm{zu}$ einer Definition von DRM-Technologie als Kontrolltechnologie, die sicherstellen soll, dass digitale Werke nur unter jenen Bedingungen, die die Informationsanbieter spezifiziert haben, genutzt werden können (vgl. Armstrong 2006, S. 1). Da solche Kontrollen im digitalen Milieu allerdings nicht $\mathrm{zu}$ 100 Prozent sicher sein können und häufig sogar leicht zu umgehen sind (und die Verfahren der Umgehung ebenso schnell wie die Inhalte selbst verbreitet werden können), wurde der rechtliche Schutz technischer Schutzmaßnahmen in Gesetzen verankert. In diesem Sinn kann von einem technisch-rechtlichen DRMParadigma gesprochen werden. ${ }^{4}$

\section{Kritik an DRM-Systemen und ihren Nebenfolgen}

Selten waren soviel öffentliches Unbehagen und aktiver Protest zu vernehmen wie bei der Einführung von DRM-Systemen zum Schutz digitaler Inhalte und der Novellierung des Urheberrechts, das unter anderem die Umgehung dieser Schutzmaßnahmen verbietet. Insbesondere in den Online-Medien wird das Thema diskutiert, wie unlängst in einer Studie des Büros für Technikfolgen-Abschätzung beim Deutschen Bundestag gezeigt wurde (Grunwald et al. 2006, S. 191 ff.). Bürgerinitiativen, Fachverbände und Verbraucherverbände stellen Manifeste auf und protestieren massiv gegen den Einsatz von DRMSystemen und entsprechende Veränderungen im Urheberrecht (BEUC 2004, Kutterer 2005, Felten 2005, Transatlantic Consumer Dia- logue 2005). Verbraucher klagen vor Gericht gegen Medienunternehmen (Helberger 2006) und gehen auf die Straße, um gegen bestimmte Anbieter zu protestieren. Rechtswissenschaftler, Informatiker, Informationswissenschaftler, Wirtschaftswissenschaftler und Wissenschaftler anderer Disziplinen kritisieren die Gesetzgebung und die Praxis der Medienunternehmen und zweifeln an der Wirksamkeit von DRM-Systemen. Einige Risiken und Nebenwirkungen dieses technisch-rechtlichen Paradigmas, die grob mit den Schlagworten Urheberrecht, Verbraucherschutz, Datenschutz, Eigentumsschutz und Interoperabilität belegt werden können, werden besonders heftig debattiert. Aus Nutzersicht verbinden sich damit folgende Kritikpunkte:

1. Urheberrecht: DRM-Systeme können in Konflikt mit dem Urheberrecht bzw. Erwartungen an das Urheberecht geraten, wenn sie z. B. Materialien „schützen“, die gar nicht oder nicht mehr (etwa weil die Schutzfrist abläuft, der Schutzmechanismus aber nicht) durch das Urheberrecht geschützt sind. Gleiches gilt, wenn DRMSysteme Nutzungen unterbinden, die durch die Schrankenbestimmungen des Urheberrechts eigentlich erlaubt wären (siehe Dreier in diesem Heft).

2. Verbraucherschutz: DRM-Systeme können auf vielfältige Weise mit dem Verbraucherschutz kollidieren, indem sie etwa Transparenzerfordernissen nicht nachkommen oder mit unfairer Vertragsgestaltung einhergehen (siehe Helberger in diesem Heft).

3. Datenschutz: DRM-Systeme können mit dem Datenschutz, dem Persönlichkeitsschutz und dem Recht auf informationelle Selbstbestimmung in Konflikt geraten, wenn z. B. ohne Zustimmung des Nutzers Inhalte personalisiert werden und Profile über Nutzungsverhalten angelegt werden (siehe Möller und Puchta in diesem Heft).

4. Eigentumsschutz: DRM-Systeme können sogar das Eigentum des Nutzers schädigen (Dateien, Software, Hardware), was zuletzt im Kontext des „Sony Rootkit Skandals“ heftig diskutiert wurde (vgl. Bohn 2005, Helberger 2006). ${ }^{5}$ 
5. Interoperabilität: DRM-Systeme können von Unternehmen strategisch eingesetzt werden, um die Interoperabilität von Formaten und Geräten zu reduzieren. Für den Kunden vermindern inkompatible proprietäre Formate und Geräte die Wahl- und die Nutzungsmöglichkeiten.

DRM-geschützte Inhalte haben überdies eine „Achillesferse“, über die zu wenig gesprochen wird: ihre begrenzte Haltbarkeit. DRM-Systeme verhindern Sekundär- bzw. Gebrauchtmärkte für digitale Inhalte (Niehüser 2005), behindern die Langzeitarchivierung, die auf die Migration von Formaten angewiesen ist (Steinke 2005), und wenn ein Anbieter seinen Dienst unversehens einstellt oder ein DRMSystem nicht weiter unterstützt und gepflegt wird, kann eine Sammlung digitaler Inhalte im $\mathrm{Nu}$ nutzlos werden. Je schneller der technologische Wandel, umso kürzer wird der Lebenszyklus DRM-geschützter Inhalte sein. Zu den subtileren Problemen gehört des Weiteren noch der ungenügend aufgeklärte Eigentumsstatus von lizenzierten digitalen Inhalten, die einem Käufer weder im Sinne eines Kaufs mit vollständiger und freier Verfügungsgewalt überlassen werden, noch wie beim Verleih oder Leasing nur temporär zur Verfügung gestellt werden (Hoeren 2003, S. 579). Unzureichend ist auch geklärt, wie sich Ansprüche von Nutzern auf medienadäquate, transformative Nutzungsformen mit der strikten Nutzungskontrolle von DRM-Systemen in Einklang bringen lassen (s. zu den rechtlichen Aspekten in diesem Zusammenhang Helberger in diesem Heft).

Bei den hier angesprochenen Nebenfolgen, die in einem weiten Sinn die Benutzerfreundlichkeit betreffen, handelt es sich noch nicht um irreversible Technikfolgen. Der berechtigten Kritik kann in diesem Stadium sowohl durch innovative und verbraucherfreundliche Geschäftsmodelle (INDICARE 2004), durch eine andere technische Gestaltung von DRM-Systemen (s. die Beiträge von Bechtold und Böhle in diesem Heft) und durch Änderungen des bestehenden Rechtsrahmens (s. die Beiträge von Dreier, Helberger und Rosenblatt in diesem Heft) begegnet werden.

\section{DRM als hochbrisantes Politikum}

Hat man einmal die kritischen Punkte identifiziert, käme es darauf an, Alternativen der Regulierung und der technischen Gestaltung zu konkretisieren und $\mathrm{zu}$ analysieren sowie ihre Nebenfolgen und Durchsetzungschancen abzuschätzen. Das ist schwierig und - soweit wir sehen - auch bisher nirgends umfassend und systematisch geleistet worden. Damit aber nicht genug: Hinter diesen offenkundigen Kritikpunkten liegen tiefer gehende gesellschaftliche Probleme und politische Auseinandersetzungen, die hier nur angedeutet werden können. Es steht zur Diskussion, ob das Urheberrecht noch dem Interessenausgleich zwischen Rechteinhabern einerseits und den Interessen von Nutzern und der Allgemeinheit andererseits dient, oder ob es sich zu einem ausschließlichen Verwertungsrecht wandelt. Ebenso strittig ist die Frage, ob DRM-Systeme und die damit verbundene Kommerzialisierung und Privatisierung von digitalen Inhalten volkswirtschaftlich rational und unter dem Gesichtpunkt der Nachhaltigkeit akzeptabel ist. ${ }^{6}$

Medien- und Informationspolitik (und folglich auch Politikberatung) in diesem Konfliktfeld werden durch den komplexen Interessenmix mit feinen Konfliktlinien und punktuellen Allianzen und durch den Bedarf an Regelungen mit tendenziell globaler Geltung natürlich erschwert. ${ }^{7}$ Die große Unsicherheit der Politik lässt sich exemplarisch an der höchst unterschiedlichen Umsetzung der Europäischen Richtlinie zum Urheberrecht in den einzelnen Mitgliedstaaten ablesen, was sich aktuell an dem Zickzack-Kurs bei der Umsetzung dieser Richtlinie in Frankreich exemplarisch zeigen lässt. Dort wurde zuerst auf die Legalisierung von Online-Tauschbörsen in Kombination mit einem pauschalen Vergütungssystem gesetzt, später auf strikteste Interoperablitätserfordernisse für DRM-Systeme. Letztendlich wurden beide Ansätze zurückgenommen. Die Sozialisten legten Verfassungsbeschwerde ein, die ironischerweise dazu führte, dass nach der Grundsatzentscheidung des französischen Verfassungsrates das Urheberrecht noch anbieterfreundlicher ausgefallen ist. ${ }^{8}$

Die konkrete Kritik der Verbraucher, die verbreitete Unsicherheit der Politik und auch der Klärungsbedarf, was das ökonomische 
Potenzial einer auf DRM-Systemen basierenden Medienwirtschaft betrifft, haben zu erheblichen Beratungsaktivitäten geführt (siehe zu den zahlreichen Konsultationsverfahren Orwat in diesem Heft). In diesem Kontext kann auch das von ITAS geleitete Projekt INDICARE verortet werden. Mit dem Schwerpunktthema möchte ITAS die Aufmerksamkeit auf die Herausforderungen für Recht, Technik und Technikfolgenabschätzung lenken.

\section{Das „INDICARE“-Projekt}

Das Projekt „INDICARE (http://www.indicare. org) - The Informed Dialogue about Consumer Acceptability of Digital Rights Management Solutions in Europe" (März 2004 bis Ende Februar 2006) ist inzwischen erfolgreich abgeschlossen worden. Das Projekt wurde bereits in TATuP vorgestellt (Orwat 2004). Hier soll nur kurz das Erreichte angesprochen werden, weil damit gleichzeitig der Ausgangspunkt dieses Themenschwerpunktes deutlich wird.

INDICARE wurde als Begleitmaßnahme im Rahmen des „eContent"-Programms (2001-2004) der Europäischen Kommission gefördert. Dieses Programm war marktorientiert, hat aber durchaus das Problem erkannt, dass ,verbraucherbezogene Aspekte bei der Verwaltung von Rechten bisher wenig berücksichtigt" wurden und hat selbst die „Schaffung eines Dialogs“ zwischen den verschiedenen Akteuren vorgeschlagen (Europäische Kommission, o. J., S. 21). Dies bildete den Ansatzpunkt für ITAS, mit seinem Konzept einer Kombination von Internet-basierten Dialogkomponenten (Website, Blog, interaktive Zeitschrift) und eigenständiger Forschung (deshalb ,informed dialogue“) auf eine entsprechende Ausschreibung zu reagieren. ${ }^{9}$

Bedingung für das Gelingen war die $\mathrm{Zu}-$ sammenstellung eines multidisziplinären Teams mit rechtlicher, technischer, wirt- schaftswissenschaftlicher und TA-Kompetenz. Dies wurde mit den weiteren Partnern (dem Institute for Information Law (IViR)) der Universität Amsterdam, dem SEARCH Laboratory der Budapest University of Technology and Economics und der Berlecon Research $\mathrm{GmbH}$ (Berlin) auch erreicht. ${ }^{10}$ Die unterschiedlichen Publikationstypen, die erstellt wurden, spiegeln die Vielfalt der wissenschaftlichen und kommunikativen Anstrengungen wider: Über zwei Jahre wurde eine begutachtete OnlineZeitschrift zum Thema, der „INDICARE Monitor", herausgegeben; es wurden zwei repräsentative europäische Konsumentenbefragungen durchgeführt, die durchaus mit den einschlägigen Umfragen des PEW / Internet Projekts zu vergleichen sind; es wurde ein Sachstandsbericht erarbeitet, der zwei Mal aktualisiert wurde und dessen Stärke darin zu sehen ist, dass das Thema DRM über seine enge Beziehung zum Urheberrecht hinaus ausgeweitet wurde auf Fragen des Verbraucherschutzes und verbraucherfreundlicher Geschäftsmodelle. Des Weiteren wurden fünf Workshops durchgeführt. Gegen Ende des Projekts wurden die Ergebnisse zielgruppenorientiert aufbereitet - zum einen für Konsumenten und Verbraucherverbände und zum anderen für die Anbieter von Inhalten. Der „Verbraucherleitfaden zum Digitalen Rechtemanagement" liegt bereits in fünf europäischen Sprachen vor. In den nächsten Monaten werden noch Ausgaben in fünf weiteren Sprachen folgen. Dazu kommen zwei „policy paper", von denen das eine versucht, DRM stärker als TA-Thema zu profilieren, und das andere, DRM-Systeme im Kontext wissenschaftlichen Publizierens und wissenschaftlicher Kommunikation zu erörtern. Letzteres ist ein Anwendungsfeld, das bislang im Vergleich zum Unterhaltungsbereich eher stiefmütterlich behandelt wurde. Einen Überblick über die unterschiedlichen Publikationen bietet die Textbox in dieser Einführung. 


\section{Zusammenstellung der im Projekt INDICARE entstandenen Publikationen}

\section{Zeitschrift INDICARE Monitor}

- INDICARE Monitor, Volume 1 (2004-2005) hrsg. v. Knud Böhle; online verfügbar unter http:/www.indicare.org/tiki-download_file.php?fileId=105

- INDICARE Monitor, Volume 2 (2005-2006) hrsg. v. Knud Böhle; online verfügbar unter http://www.indicare.org/tiki-download_file.php?fileId=178

- Die einzelnen Artikel sind online verfügbar unter http://www.indicare.org/monitor

INDICARE State-of-the-Art-Berichte

- INDICARE State-of-the-Art Report (2004): Digital Rights Management and Consumer Acceptability. A Multi-Disciplinary Discussion of Consumer Concerns and Expectations, hrsg. v. Natali Helberger, verfasst von Nicole Dufft, Stef van Gompel, Kristóf Kerényi, Bettina Krings, Rik Lambers, Carsten Orwat, und Ulrich Riehm, Dezember 2004, online verfügbar unter http://www.indicare.org/tiki-download_file.php?fileId=60

- INDICARE State-of-the-Art Report, First Supplement (2005): Digital Rights Management and Consumer Acceptability. A Multi-Disciplinary Discussion of Consumer Concerns and Expectations, hrsg. v. Natali Helberger, verfasst von Nicole Dufft, Margreet Groenenboom, Kristóf Kerényi, Carsten Orwat, und Ulrich Riehm, Ma1 2005, online verfügbar unter http://www.indicare.org/tiki-download_file.php?fileId=111

- INDICARE State-of-the-Art Report, Second Supplement (2006) (unv. Manuskript)

INDICARE Umfragen

- INDICARE Consumer Survey (2005): Digital Music Usage and DRM, verfasst von Nicole Dufft, Andreas Stiehler, Danny Vogeley und Thorsten Wichmann, Mai 2005, online verfügbar unter http://www.indicare.org/tiki-download_file.php?fileId=110

- INDICARE Consumer Survey (2006): Digital Video Usage and DRM, verfasst von Nicole Dufft, Philipp Bohn, Andreas Stiehler, und Thorsten Wichmann, Februar 2006, online verfügbar unter http://www.indicare.org/tiki-download_file.php?fileId=170

INDICARE Workshop Berichte

- INDICARE Workshop Report (2004), Business Models for Mobile Music and DRM, verfasst von Kristóf Kerényi, Sep 2004, online verfügbar unter http://www.indicare.org/tiki-download_file.php?fileId=49

- INDICARE Workshop Report (2005): E-Payment and DRM for Digital Content, verfasst von Ernö Jeges and Kristóf Kerényi, Feb 2005, online verfügbar unter http://www.indicare.org/tiki-download_file.php?fileId=98

- INDICARE Workshop Report (2005): Fair DRM Use, verfasst von Mara Rossini and Natali Helberger, May 2005, online verfügbar unter http://www.indicare.org/tiki-download_file.php?fileId=146

- INDICARE Workshop Report (2006): Digital Rights Management in Public Science, verfasst von Carsten Orwat, online verfügbar unter http://www.indicare.org/tiki-download_file.php?fileId=176

- INDICARE Workshop Report (2006): Human Factors of Digital Rights Management, verfasst von Kristóf Kerényi, online verfügbar unter http://www.indicare.org/tiki-download_file.php?fileId=193

INDICARE Leitfäden

- INDICARE Content Providers' Guide to DRM (2006), verfasst von Philipp Bohn unter Mitarbeit von Carsten Orwat und Knud Böhle, März 2006, online verfügbar unter http://www.indicare.org/user-guide/

- INDICARE Verbraucherleitfaden zum Digitalen Rechtemanagement (2006) verfasst von Margreet Groenenboom und Natali Helberger unter Mitarbeit von Carsten Orwat, Martien Schaub und Mathias Spielkamp, online verfügbar unter http://www.indicare.org/tiki-download_file.php?fileId=194

- Der Verbraucherleitfaden liegt insgesamt in zehn europäischen Sprachen vor; online verfügbar unter http://www.indicare.org/tiki-page.php?pageName=ConsumerGuide

INDICARE Policy Paper

- INDICARE First Policy Paper (2006): Digital Rights Management in Scientific Publishing and Communication, verfasst von Carsten Orwat, Karlsruhe 2006 (unv. Manuskript)

- INDICARE Second Policy Paper (2006): Digital Rights Management Systems - A Whiff of Technology Assessment, verfasst von Knud Böhle, Karlsruhe 2006 (unv. Manuskript) 


\section{Zu den Beiträgen des Schwerpunkts}

Ergebnisse des INDICARE-Projekts, die unter der Perspektive rechtlicher, politischer und technischer Gestaltungsoptionen von besonderem Interesse sind, werden in diesem Schwerpunkt den Lesern der TATuP vorgestellt. Dabei gehen die Beiträge über das aus den Projektpublikationen Bekannte hinaus. Zusätzlich zu den INDICARE-Partnern, die hier zu Wort kommen (ITAS, IvIR, Berlecon), haben wir die ausgewiesenen Experten Thomas Dreier, Stefan Bechtold und Bill Rosenblatt, die INDICARE unter anderem durch ihre Beiträge für den INDICARE-Monitor unterstützt haben, als Autoren für den Schwerpunkt gewinnen können. Hinzugenommen haben wir außerdem einen Beitrag von Jan Möller und Stefan Puchta, deren Projekt „Privacy4DRM“ über den Projektträger Innovations- und Technikanalyse (ITA) vom Bundesministerium für Forschung und Technologie gefördert wurde. ${ }^{11}$

Die Anordnung der Beiträge ist so vorgenommen worden, dass eingangs die einschlägigen rechtlichen Entwicklungen in Deutschland und den USA dargestellt werden. Im Anschluss werden dann die Risiken und Nebenwirkungen von DRM-Systemen in punkto „Datenschutz“ und "Verbraucherschutz" behandelt sowie Verbesserungsmöglichkeiten vorgeschlagen. Die Erkenntnisse der repräsentativen INDICAREKonsumentenbefragungen ergänzen diesen Themenblock. In den folgenden zwei Beiträgen wird die Frage nach der Gestaltung von DRMSystemen untersucht - zum einen aus dem Blickwinkel rechtlicher Technikgestaltung und zum anderen mit dem analytischen Ziel, alternative Gestaltungsoptionen $\mathrm{zu}$ identifizieren und diese leitenden (,wissensordnungspolitischen") Orientierungen zuzuordnen. In dem abschließenden Beitrag wird die Bedeutung von Konsultationsprozessen in der politischen Gestaltung von DRM in Europa nachgezeichnet. Dieser Beitrag schließt mit der Frage, ob angesichts der vielfältigen Politikbereiche, die mit DRM befasst sind und der Ausbreitung von DRM-Systemen in einer Vielzahl von Anwendungsfeldern und Wirtschaftszweigen, nicht umfassende TA-Studien dringend notwendig wären. Im Folgenden werden die einzelnen Beiträge kurz vorgestellt.
Wer von DRM redet, kann vom Urheberrecht nicht schweigen. Der Beitrag von Thomas Dreier, Leiter des Zentrums für angewandte Rechtswissenschaft (ZAR) an der Universität Karlsruhe, führt präzise und kritisch in das Thema ein. Er erläutert eingangs, was es mit dem rechtlichen Schutz gegen die Umgehung technischer Schutzmaßnahmen im Urheberrecht auf sich hat. Im Anschluss analysiert er, welche Chancen und Risiken mit der gegenwärtigen Lösung unter dem Aspekt der Steuerung - sowohl des technischen Innovationsverhaltens als auch des Verhaltens von Nutzern urheberrechtlich geschützter Werke und Leistungen - verbunden sind. In einem Ausblick weist er auf ein wichtiges Zukunftsthema hin: auf die über den Medienbereich hinausgehende Bedeutung des "digitalen Rechtemanagements“.

Im zweiten Beitrag kommt einer der ausgewiesenen DRM-Experten der USA zu Wort. Bill Rosenblatt, Buchautor, Berater und Betreiber des Online-Dienstes DRM Watch ${ }^{12}$, geht in seiner Policy Analysis zunächst auf den rechtlichen Schutz gegen die Umgehung technischer Schutzmaßnahmen im „US Copyright Law" ein und arbeitet vergleichend die Unterschiede zum europäischen Recht heraus. Im Anschluss geht es um neuere Regulierungsanstrengungen bezogen auf die Spannung zwischen dem „fair-use“-Prinzip und DRM, auf Haftungsansprüche gegen Dritte (,secondary infringement liability") und Vorschriften, die den Einsatz von DRM-Systemen obligatorisch machen könnten (,mandatory DRM“). Für ein „Assessment" von DRM schlägt er vor, die Auswirkungen auf die Balance im Copyright, die Nebenwirkungen für Verbraucher und die Frage nach dem gesamtwirtschaftlichen Nutzen von DRM zu untersuchen.

Der Schutz der Privatsphäre ist eines der wichtigen Rechtsgüter, das durch DRM-Systeme bedroht wird. Darum hatten wir das Projektteam um Rüdiger Grimm und Johann Bizer, die das vom Bundesministerium für Bildung und Forschung (BMBF) geförderte Projekt „Privacy 4DRM“" leiteten, gebeten, die wichtigsten Ergebnisse für uns aufzubereiten. Diese Aufgabe übernahmen schließlich Jan Möller (Unabhängigen Landeszentrum für Datenschutz Schleswig-Holstein) und Stefan Puchta (FraunhoferInstitut für Digitale Medientechnologie). Der Beitrag erläutert die rechtlichen Anforderungen 
an DRM-Systeme und führt den empirischen Nachweis, dass diesen Anforderungen oft nicht entsprochen wird. Insbesondere mangele es an hinreichender Transparenz und einer datensparsamen Technikgestaltung. Der Artikel schließt mit Handlungsempfehlungen und Hinweisen auf weiteren Forschungsbedarf. Datenschutz-Gütesiegel für DRM-Produkte werden als eine interessante Möglichkeit gesehen, mehr Transparenz $\mathrm{zu}$ erreichen. Forschungsbedarf wird vor allem für den DRM-Einsatz im Bildungsbereich gesehen (eLearning, Bibliotheken, Vertrieb von Lehrmaterialien).

Natali Helberger vom renommierten Institute for Information Law (IViR) der Universität Amsterdam, das als Partner im INDICAREProjekt juristische Expertise einbrachte, entwickelt in ihrem Beitrag nicht mehr und nicht weniger als eine rechtliche DRM-Agenda mit dem Ziel, die Interessen der Verbraucher besser zur Geltung zu bringen. Die Autorin kann zeigen, dass das Urheberrecht die Interessen der Verbraucher nicht hinreichend schützen kann, da es nicht auf der Ebene von Nutzungen operiert (wie DRM-Systeme). Weiterhin enthalte es auch kein zeitgemäßes Konzept des „Nutzers“ digitaler Inhalte und könne schon vom Anspruch her nicht alle Verbraucheraspekte abdecken. Vor diesem Hintergrund werden dann Optionen für die Gestaltung des Verbraucherschutzes im Hinblick auf digitale Medien aufgezeigt, wobei vier Ansätze herausgearbeitet werden: der Weg über die Verbraucherautonomie; eine innovations- und verbraucherfreundliche Reinterpretation des Urheberrechts, eine Weiterentwicklung des allgemeinen Verbraucherschutzrechtes und, was Natali Helberger für besonders viel versprechend hält, sektorspezifische Regeln zum Schutz der Verbraucherinteressen. In diesem Punkt ließe sich durchaus vom Umwelt- oder Telekommunikationsrecht lernen.

Im nächsten Beitrag stellt Philipp Bohn (Berlecon Research $\mathrm{GmbH}$ ) die wichtigsten Ergebnisse der zwei von Berlecon im Rahmen des INDICARE Projekts durchgeführten Verbraucherumfragen vor. Gerade im Kontrast zu den öffentlichen Protesten gegen DRM, die der Autor in seinem Beitrag einleitend aufgreift, sind die Ergebnisse der repräsentativen Erhebungen höchst interessant: Es konnte z. B. festgestellt werden, dass vielen Konsumenten das Urheberrecht kaum bekannt ist oder sie sich kaum darum scheren. Gleichzeitig wissen auch nur Wenige, was DRM überhaupt ist und welche Folgen damit verbunden sind. Abschließend erörtert der Autor, ob politischer Handlungsbedarf besteht. Sein Ergebnis lautet: Aufklärung besonders Jugendlicher über das Urheberrecht erscheint angebracht, sollte aber auch Informationen über illegale Geschäftspraktiken mancher Anbieter umfassen; mehr Transparenz durch Gütesiegel wird begrüßt, eine stärkere Intervention des Gesetzgebers aber eher als problematisch erachtet.

Oft wird über der Kritik an DRM-Systemen die Frage vergessen, ob DRM-Systeme nicht auch ganz anders gestaltet werden könnten, so dass die Kritik gegenstandslos würde. Stefan Bechtold, heute am Max-Planck-Institut zur Erforschung von Gemeinschaftsgütern, der sich schon 2002 durch seine rechtswissenschaftliche Dissertation zu DRM einen Namen gemacht hat, befasst sich hier mit der rechtlichen Technikgestaltung. Er betont die Möglichkeiten einer an rechtlichen und politischen Werten orientierten Gestaltung von DRMSystemen und skizziert die Umrisse des dazu entstehenden interdisziplinären Forschungsgebiets. Dieser Beitrag wurde bereits 2004 im INIDICARE Monitor veröffentlicht, liegt aber nun erstmals auf deutsch und in einer leicht überarbeiteten Fassung vor.

Angeregt durch die Arbeit Bechtolds, wird in dem folgenden Beitrag von Knud Böhle (ITAS) genereller nach den Optionen der Gestaltung von DRM-Systemen gefragt und versucht, das Thema für die sozialwissenschaftliche Technikforschung zu erschließen. Nach einigen einführenden Schritten, in denen DRM-Systeme definiert und die unterschiedlichen Anwendungsfelder skizziert werden, werden Optionen der Technikgestaltung unterschieden, die sich entweder vorrangig am Verwertungsinteresse oder am Interessenausgleich orientieren. Dazu kommt eine dritte Option, die wegen ihrer Bedeutung für die politische Diskussion einbezogen wird. Es handelt sich dabei um einen Ansatz, der auf Interessenausgleich ohne den Einsatz von DRM-Systemen zielt. Der Artikel schließt mit einigen Hinweisen auf offene Forschungsfragen.

Der Schwerpunkt wird durch einen Beitrag von Carsten Orwat (ITAS), abgeschlossen, der sich mit Konsultationsverfahren befasst, die mit 
der Einführung von DRM-Systemen und insbesondere der Gestaltung des rechtlichen Rahmens verbunden waren (und zum Teil noch sind). Die zahlreichen Konsultationsverfahren und Projekte auf europäischer Ebene sowie nationale Aktivitäten in England, Dänemark, Norwegen und Deutschland werden detailliert aufgezeigt. Im Anschluss an die Präsentation der Verfahren und Projekte werden der mögliche „Impact“ der Projekte und die Probleme bei der Umsetzung der Ergebnisse und Empfehlungen diskutiert. Abschließend wird konstatiert, dass bisher nur wenige TA-Projekte zu diesem Thema durchgeführt wurden, dass aber gerade jetzt wegen der zunehmenden Komplexität (zunehmende Zahl der beteiligten Rechtsgebiete, Politikfelder und Anwendungsfelder) einiges für umfassende und abwägende Analysen sprechen würde.

\section{Anmerkungen}

1) Neuere Zahlen, mit denen auch Trends aufgezeigt werden könnten, sind uns nicht bekannt.

2) Vgl. Coyle 2005 für den Bibliotheksbereich, für den Wissenschaftsbereich insgesamt siehe INDICARE 2006a und INDICARE 2006b.

3) Diese Sichtweise findet sich ausgebaut bei Beninger (1986), der in seinem Buch über die „Control Revolution“ die These vertritt, dass die wachsende Komplexität der Industriegesellschaft eine „control crisis“ erzeugt hatte, die zur Entwicklung unterschiedlicher neuer Kontrolltechnologien führte, von denen eine die Computertechnologie war.

4) Siehe dazu auch Ünlü: „In fact, the technological and legal approaches provide mutual support for one another, thereby constituting a protective unity. Technology prevents infringements, while legal measures provide deterrents regarding circumvention of the technology.“ (Ünlü 2005)

5) Gelegentlich werden sogar Stimmen laut, die in der Schädigung des Eigentums qua ,technical self-help-measures“" (man könnte auch sagen qua Selbstjustiz) eine Möglichkeit sehen, auf illegales Kopieren zu reagieren (Böhle 2004).

6) Grassmuck diskutiert diese Fragestellung unter dem Stichwort der ,informationellen Nachhaltigkeit“ und Kuhlen unter dem der „Wissensökologie“ (Grassmuck 2002, insbes. S. 162; Kuhlen 2004, S. 263 ff.).

7) Weber und Haug (2003) haben einmal eine differenzierte Durchsicht der Akteure und Interessen mit Bezug auf Online-Tauschbörsen vorgelegt. Bei den Konfliktlinien ist etwa an die unterschiedlichen Interessen der Medien- industrie einerseits und der Geräteindustrie und der Netzwerkbetreiber andererseits zu denken, bei den Allianzen etwa an die Ablehnung von Pauschalabgaben von Seiten der ITIndustrie und der Verbraucherverbände, die in diesem Punkt nicht mit anderen zivilgesellschaftlichen Gruppen übereinstimmen, die ihnen ansonsten nahe stehen.

8) Siehe dazu http://www.heise.de/newsticker/ meldung/print/74968 und http://www.heise.de/ newsticker/meldung/76208.

9) Dabei konnte ITAS auf Erfahrungen mit diesem Ansatz in anderen Projekten aufbauen, insbesondere dem Projekt „Elektronische Zahlungssysteme"(http://www.itas.fzk.de/deu/PROJEKT/pez.h tm) und dem „electronic Payment Systems Observatory - ePSO“" (inzwischen http://epso.intra soft.lu/files/archives.html).

10) Im ITAS waren Carsten Orwat (als Koordinator des Projekts) und Knud Böhle (als Herausgeber des „INDICARE Monitor") hauptsächlich für das Projekt zuständig. Ulrich Riehm und Bettina Johanna Krings arbeiteten an dem Projekt vor allem in der Anfangsphase mit, während Michael Rader laufend die Redaktion der Zeitschrift unterstützte.

11) Eine ausführliche Besprechung des Projektes „Privacy4DRM“ findet sich im INDICARE Monitor (Böhle 2006).

12) Die Website dieses Online-Dienstes ist zu finden unter http://www.drmwatch.com/.

\section{Literatur}

Armstrong, T.K., 2006: Digital Rights Management and the Process of Fair Use. Online verfügbar bei SSRN unter der Adresse http://ssrn.com/abstract= 885371 [überprüft am 28.7.2006]; (demnächst in Harvard Journal of Law \& Technology 20/1 <2006>)

Beninger, J.R., 1986: The Control Revolution: Technological and Economic Origins of the Information Society. Cambridge, Massachusetts: Harvard University Press

BEUC, 2004: Digital Rights Management (DRM) BEUC Position paper, X/025/2004, Brussels, 15.9.2004; online verfügbar unter http://www.beuc. org/Content/Default.asp?PageID=103 [überprüft am 28.7.2006]

Böhle, K., 2004: Of vanishing media and copyright enforcement by destruction. In: INDICARE Monitor Vol. 1, No 2, July 2004; online verfügbar unter http://www.indicare.org/tiki-read_article.php? articleId=29 [überprüft am 28.7.2006]

Böhle, K., 2006: Research into user-(un)friendly DRM. A review. In: INDICARE Monitor Vol. 2, No 12, February 2006; online verfügbar unter 
http://www.indicare.org/tiki-read_article.php? articleId=174 [überprüft am 28.7.2006]

Bohn, Ph., 2005: Intrusive DRM: The cases of Sony BMG, StarForce and Microsoft. In: INDICARE Monitor Vol. 2, No 9, November 2005; online verfügbar unter http://www.indicare.org/tiki-read article.php?articleId=155 [überprüft am 28.7.2006]

Coyle, K., 2005: The role of digital rights management in library lending. In: INDICARE Monitor, Vol. 2, No 2, April 2005; online verfügbar unter http://www.indicare.org/tiki-read article.php? articleId=95 [überprüft am 28.7.2006]

Europäische Kommission, o. J.: eContent Arbeitsprogramm 2003-2004. Luxemburg: Europäische Kommission

Felten, E., 2005: DRM and Public Policy. Communications of the ACM, 48/7 (2005), S. 112

GfK / BPW, 2006: Brenner Studie 2006. Study on behalf of the Bundesverbandes der phonographischen Wirtschaft e.V. (BPW). Berlin: BPW/IFPI; online verfügbar unter http://www.ifpi.de/wirtschaft/brenner studie2006.pdf [überprüft am 28.7.2006]

Grassmuck, V., 2002: Freie Software. Zwischen Privat- und Gemeineigentum. Bonn: Bundeszentrale für politische Bildung

Grunwald, A; Banse, G.; Coenen, Chr. et al., 2006: Netzöffentlichkeit und digitale Demokratie. Tendenzen politischer Kommunikation im Internet. Berlin: edition sigma, Studien des Büros für Technikfolgen-Abschätzung, Bd. 18

Hansen, S., 2003: CD-Schutz kontra Verbraucherschutz. In: c't 7/2003, S. 136; online verfügbar unter http://193.99.144.85/ct/03/07/136/ [überprüft am 31.7.2006]

Helberger, N., 2006: Christophe R. vs Warner Music: French court bans private-copying hostile DRM. In: INDICARE Monitor Vol. 2, No 12, February 2006; online verfügbar unter http://www.indicare.org/tikiread_article.php?articleId $=180$ [überprüft am 28.7.2006]

Hoeren, Th., 2003: Copyright Dilemma: Access Right as a Postmodern Symbol of Copyright Deconstruction. In: Becker, E. et al. (Hg.): Digital Rights Management. Technological, Economic, Legal and Political Aspects. Berlin: Springer, S. 574-586

INDICARE State-of-the-Art Report, 2004: Digital Rights Management and Consumer Acceptability. A Multi-Disciplinary Discussion of Consumer Concerns and Expectations, hrsg. v. Natali Helberger, verfasst von Nicole Dufft, Stef van Gompel, Kristóf Kerényi, Bettina Krings, Rik Lambers, Carsten Orwat, und Ulrich Riehm, Dezember 2004; online verfügbar unter http://www.indicare.org/tiki-download_ file.php?fileId $=60$ [überprüft am 28.7.2006]
INDICARE First Policy Paper, 2006a: Digital Rights Management in Scientific Publishing and Communication, verfasst von Carsten Orwat, Karlsruhe 2006 (unv. Manuscript)

INDICARE Workshop Report, 2006b: Digital Rights Management in Public Science, verfasst von Carsten Orwat, online verfügbar unter http://www.indicare. org/tiki-download_file.php?fileId=176 [überprüft am 28.7.2006]

Kuhlen, R., 2004: Informationsethik. Konstanz: UVK Kutterer, C., 2005: Some of the reasons for BEUC's Campaign on Consumers' Digital Rights. In: INDICARE Monitor, Vol. 2, No 10, December 2005; online verfügbar unter http://www.indicare.org/tikiread_article.php?articleId $=162$ [überprüft am 28.7.2006]

Niehüser, L., 2005: Sekundärmärkte für digitale Medien auf Basis des Digital Rights Management. Rechtliche und technologische Voraussetzungen sowie ökonomische Implikationen. Aachen: Shaker

Orwat, C., 2004: INDICARE: Consumers, Users and Digital Rights Management. In: Technikfolgenabschätzung Theorie und Praxis 13(2004)1, S. 155-158; online verfügbar unter http://www.itas.fzk.de/tatup/ 041/itas-news.htm [überprüft am 28.7.2006]

Shapiro, A.L., 1999: The Control Revolution. How the Internet is putting individuals in charge and changing the world we know. New York: Public Affairs

Steinke, T., 2005: National libraries, preservation and digital rights management. Interview von Knud Böhle mit Tobias Steinke. In: INDICARE Monitor, Vol. 2, No. 5, May 2005 online verfügbar unter http://www.indicare.org/tiki-read_article.php? articleId=107 [überprüft am 28.7.2006]

Transatlantic Consumer Dialogue (TACD), 2005: Resolution on Digital Rights Management. Doc No. IP-01-05. April 2005; online verfügbar unter http://www.tacd.org/docs/?id=275 [überprüft am 28.7.2006]

Ünlü, V., 2005: Profitable piracy and content degradation - An alternative to strong content protection. In: INDICARE Monitor, Vol. 2, No. 8, October 2005; online verfügbar unter http://www.indicare.org /tiki-read_article.php?articleId=148 [überprüft am 28.7.2006]

Weber, K.; Haug, S., 2003: Konfrontation oder Kompromiss? Empirische Befunde und ethische Überlegungen zu Urheberrechtskonflikten. In: Ethica, $11 / 2$ (2003), S. 163-184 